\title{
Typologie et rentabilité des systèmes de production laitière à Linguère au Sénégal
}

\author{
Sèdjolo Stève DASSOU ${ }^{12^{*}}$, Idrissa WADE $^{2}$ et Codjo Emile AGBANGBA ${ }^{3}$ \\ ${ }^{1}$ Centre International de Recherche et de Formation en Sciences Sociales (CIRFOSS), \\ BP : 2540, Abomey-Calavi, Bénin. \\ ${ }^{2}$ Ecole Nationale Supérieure d'Agriculture, Université de Thiès, Sénégal. \\ ${ }^{3}$ Ecole Polytechnique d'Abomey-Calavi, Université d'Abomey-Calavi, Bénin. \\ *Auteur correspondant, E-mail : stevedassou@yahoo.fr ; Tél : (+229) 97639010
}

\section{REMERCIEMENTS}

Ce travail a été financé par l'Organisation des Nations Unies pour le Développement Industriel (ONUDI) dans le cadre du projet de promotion du développement économique local de la région de Louga au Sénégal.

\section{RESUME}

La connaissance des systèmes de production laitière est un atout pour une intervention efficace dans le sous-secteur de l'élevage. C'est dans ce cadre que cette étude vise à faire une typologie et une analyse de la rentabilité des systèmes de production laitière à Linguère au Sénégal. La méthode «Boule de neige » a été utilisée pour l'échantillonnage des éleveurs. L'Analyse des Correspondances Multiples (ACM) suivie d'une Classification Hiérarchique Ascendante (CHA) ont permis de réaliser la typologie. L'étude a fait ressortir trois (03) types de systèmes de production laitière qui se différencient par l'effectif des femelles du troupeau, le nombre de vaches en lactation, la production laitière, la distance séparant l'exploitation de l'unité de transformation locale la plus proche, le choix du produit vendu, l'orientation commerciale et l'objectif visé par l'élevage. En saison sèche, les marges brutes sont négatives à cause des fortes dépenses en compléments alimentaires. Toutefois, il n'y a pas de différence significative entre les marges brutes des éleveurs des systèmes 1 et 2 . En saison pluvieuse, les marges brutes sont positives du fait de l'absence de la complémentation. En effet, elles sont de $1047 \pm 165 \mathrm{~F} \mathrm{CFA} /$ vache/jour et de $700 \pm 66 \mathrm{~F} \mathrm{CFA/vache/jour,}$ respectivement pour les éleveurs des systèmes 1 et 2 . Les éleveurs du système 3 sont totalement orientés vers l'autoconsommation du lait.

(C) 2017 International Formulae Group. All rights reserved.

Mots clés : Éleveurs, vache, lait, classification, rentabilité, Sénégal. 


\title{
Typology and profitability of dairy production systems in Linguère, Senegal
}

\begin{abstract}
The understanding of dairy production systems is an asset for effective interventions in the livestock sub-sector. The objective of this research is to make a typology and to analyze the profitability of dairy production systems in Linguère, Senegal. The "Snowball" method was used to sample pastoralists. Multiple Correspondence Analysis (MCA) followed by an Ascending Hierarchical Classification (AHC) helped to realize the typology. The study showed three types of dairy production systems, different one from another by the number of females in the herd, the number of lactating cows, dairy production, the distance separating the farm from the closest local processing unit, the choice of the product sold, the business direction and the objective of the husbandry. In dry season, gross margins are negative due to high expenditure on food supplements. But there is no significant difference between the gross margins of pastoralists in systems 1 and 2. In rainy season, gross margins are positive due to the absence of supplementation. Indeed, it is $1,047 \pm 165$ FCFA / cow / day and $700 \pm 66$ FCFA / cow / day respectively for farmers in systems 1 and 2. Pastoralists in system 3 are totally oriented towards self-consumption of milk.

(C) 2017 International Formulae Group. All rights reserved.
\end{abstract}

Keywords: Pastoralists, cow, milk, classification, profitability, Senegal.

\section{INTRODUCTION}

Le lait est un produit stratégique pour les systèmes de production en Afrique subsaharienne. En effet, il est utilisé comme matière première pour l'élevage mais aussi pour l'alimentation des éleveurs et agroéleveurs (Holloway et al., 2000). Au Sénégal, le lait est le second contributeur au chiffre d'affaire du secteur primaire après les produits avicoles avec 9,7\% (DPEE, 2011). Il est utilisé comme rafraichissement mais également mélangé aux céréales sous forme de bouillie (de mil, de maïs...) au petit déjeuner ou au repas du soir (Dia, 2013). Sa production a connu une augmentation de 27,14\% entre 2010 à 2014, passant de 155823 tonnes à 198111 tonnes (FAOSTAT, 2014).

Malgré l'augmentation de la production ces dernières années, elle couvre à peine $35 \%$ des besoins de consommation annuelle de la population (Dia, 2009). Aujourd'hui, dans le but de permettre l'accès des consommateurs urbains à faible pouvoir d'achat aux produits laitiers de qualité et à bon marché, les responsables politiques facilitent leurs importations (Pinaud, 2014).
Par ailleurs, les niveaux de consommation de lait et produits laitiers de la population restent encore relativement faibles : 40 équivalents litres par habitant par an en 2009 , dont $77 \%$ sous forme de lait en poudre (ANSD, 2010), alors que la norme recommandée est de 90 litres par habitant par an (Corniaux, 2015).

Cette situation démontre l'incapacité des producteurs de lait sénégalais à satisfaire la demande de la population qui ne cesse d'augmenter. Or le pays dispose de plusieurs zones agro-écologiques, dont la zone sylvopastorale, ayant des potentiels réels de production laitière. En effet, la zone sylvopastorale participe à environ $40 \%$ de la production laitière nationale à partir du système pastoral (Diao, 2009). Le département de Linguère, faisant objet de cette étude, en fait partie intégrante. Malgré ce potentiel, la transhumance, la non spécialisation des éleveurs dans la production laitière, le faible potentiel génétique laitier des bovins zébu de races locales, la dépendance vis-à-vis du pâturage naturel et une mauvaise gestion des animaux sont autant de facteurs qui entravent la production laitière et rendent 
incertain l'avenir du lait local au Sénégal (Corniaux et al., 2012 ; Marshall, 2016 ; Kassa et al., 2016).

Pour valoriser les ressources locales et réduire la facture laitière, le gouvernement en partenariat avec l'Organisation des Nations Unis pour le Développement Industriel a initié un certain nombre de projet dont le « Projet de promotion du développement économique local dans la région de Louga ». L'objectif global de ce projet est de contribuer à la réduction de la pauvreté et à la promotion du développement durable dans la région de Louga. Pour ce faire, la chaîne de valeur du lait, entre autres a été choisie pour impulser le développement économique local dans le département de Linguère. Pour une efficacité des interventions, il convient de tenir compte des différents systèmes de production (Mbetid-Bessane et al., 2002). Une meilleure connaissance des systèmes de production laitière du département est nécessaire pour connaitre les groupes cibles sur lesquels il faut agir pour améliorer et augmenter la production laitière. Cependant, aucune typologie des systèmes de production laitière à l'échelle du département ni de la région n'existe dans la littérature. Les travaux existant ne font état que de typologie à l'échelle nationale basée uniquement sur la nature et la quantité d'intrants apportés lors de la production (Dia, 2013). Il s'avère donc essentiel pour la réussite de ce projet, d'analyser les systèmes de production laitière de Linguère suivant des critères outre que la nature et la proportion d'intrants utilisés pour la production.

Cette étude vise à faire une typologie et une analyse de la rentabilité des systèmes de production laitière dans le département de Linguère afin de mieux aider les acteurs au développement à des interventions ciblées. L'existence d'une diversité de systèmes de production laitière dans le département de
Linguère a été l'hypothèse émise dans ce travail.

\section{MATERIEL ET METHODES \\ Zone d'étude}

Département de la région de Louga, Linguère est situé entre les Latitudes $15^{\circ} 21^{\prime} 32$ Nord et les Longitudes 159'29 Ouest. Il couvre une superficie de $15375 \mathrm{~km}^{2}$, soit plus du tiers du territoire national.

Le département a un climat de type sahélien continental, semi-aride, monomodal avec une saison sèche allant d'octobre à juin et une saison pluvieuse (ARD, 2013). La précipitation moyenne de 2002 à 2014 est de l'ordre de 419,59 $\mathrm{mm}$ avec une variation interannuelle (Ndione et al., 2014). La végétation se présente en saison des pluies sous forme d'un tapis continu de plantes herbacées piquetées d'arbres et arbustes. Mais le reste de l'année, les sols sont nus et particulièrement vulnérables à l'érosion éolienne et aux UV solaires. On y trouve des espèces comme Aristida mutabilis, Eragrotis tremula, Boscia senegalensis, Andropogon gayanus et Aristidia longiflora, etc.

L'élevage constitue l'activité principale du département, c'est la raison pour laquelle une très grande partie de la superficie du département (plus de 90\%) est réservée pour cette activité (ARD, 2013).

\section{Echantillonnage et collecte de données}

L'étude a été réalisée grâce à des enquêtes auprès des producteurs de lait. Etant donné qu'il n'existe pas de liste exhaustive de producteurs de lait dans le département de Linguère, les premières actions ont consisté à cibler les communes dans lesquelles, on note la présence d'unités de transformation ou de centres de collecte qui pouvaient nous mettre en contact avec leurs fournisseurs. Dans les communes où ces unités sont inexistantes, ce sont les agents vétérinaires qui ont joué le rôle 
de facilitateurs en nous orientant vers les éleveurs qui produisent du lait.

Au total, 112 éleveurs ayant produit du lait durant les deux saisons (saison pluvieuse et saison sèche) ont participé au sondage. La méthode «boule de neige » a été utilisée principalement pour cette étude. Elle s'est basée sur le réseau social d'un premier contact qui a guidé l'équipe à son prochain contact (Goodman, 1961).

Le questionnaire a couvert les saisons sèche et pluvieuse et plusieurs domaines pour comprendre au mieux les systèmes de production et de valorisation du lait. Les informations collectées ont porté sur la structure de la famille (l'âge de l'enquêté, le nombre d'actifs de l'exploitation, le niveau de scolarisation, etc.), la structuration du troupeau (le cheptel, l'effectif des métisses, le nombre de vaches en lactation durant les deux saisons, etc.), le système alimentaire (il a été détaillé sur le plan de la quantité et des variations suivant les saisons), le fonctionnement du système laitier (le nombre de traite par jour, les produits commercialisés, le mode de payement du lait commercialisé, etc.). Enfin, la production totale journalière de la ferme a été estimée suivant la formule ciaprès :

$$
\mathbf{P T J}=\mathbf{P J V} \times \mathbf{N V L}
$$

Avec : PTJ $=$ Production Totale Journalière $\mathrm{PJV}=$ Production moyenne Journalière par Vache

Lactation

$$
\text { NVL }=\text { Nombre de Vaches en }
$$

Les quantités journalières vendues et autoconsommées ont également été estimées.

Outre les variables structurelles et fonctionnelles, des variables économiques permettant de calculer les charges et les revenus ont été intégrées dans le questionnaire puis dans la base des données pour l'analyse des performances économiques des différents systèmes de production laitière. Ainsi, le revenu brut journalier a été calculé suivant la formule ci-après :

$$
\mathbf{R B J}=\mathbf{P T J} \times \mathbf{P V M}
$$

Avec : RBJ = Revenu Brut Journalier

$\mathrm{PTJ}=$ Production Totale Journalière

PVM $=$ Prix de Vente Moyen

Le coût de l'aliment a été calculé suivant les quantités déclarées achetées et distribuées. La main d'œuvre familiale a été introduite dans les calculs suivant son coût d'opportunité. Les autres dépenses (eau, soins vétérinaires, le transport) ont également été estimées. Tous les calculs de coût effectués pour le système de production laitier n'ont pris en compte que les vaches en lactation, ce qui a permis d'évaluer le coût de la ration journalière du système de production laitier.

\section{Analyses statistiques}

Les données de l'enquête ont été saisies dans le logiciel Sphinx (version 4.5.0.19) avant d'être importées dans le logiciel $\mathrm{R}$ (version 3.1.0) pour les analyses statistiques. Les analyses suivantes ont été effectuées (Youssao et al., 2013 ; Housson et al., 2016 ; Faihun et al., 2017) :

- une Analyse des Correspondances Multiples (ACM) qui a permis d'obtenir une représentation des exploitations sous forme de projections sur des plans définis par des axes factoriels.

- une Classification Ascendante Hiérarchique (CAH) réalisée sur les coordonnées des exploitations dans le plan factoriel formé par les deux premières dimensions de l'AFCM.

- des statistiques descriptives (fréquences, moyennes, variances, erreur-type, etc.) sur les principales variables quantitatives caractéristiques des systèmes de production laitière identifiés.

- un test de Kruskall Wallis a permis de comparer les systèmes les uns par rapport aux autres suivant les variables quantitatives les 
plus caractéristiques. Le seuil de significativité retenu est de 5\%.

\section{RESULTATS}

\section{Profil socio-démographique des éleveurs}

La production du lait de vache dans le département de Linguère est assurée par des pasteurs (32\% des enquêtés) ou des agropasteurs $(68 \%$ des enquêtés). Ces éleveurs sont en majorité des peulhs (94\% des enquêtés). Les systèmes de production appartiennent aux modèles d'exploitation familiale patriarcale. Les décisions de production et de vente (achat d'animaux, achat d'aliments, vaccination, vente, etc.) concernant l'élevage en général sont prises par le chef du ménage; mais celles touchant en particulier la sphère post production (traite et vente du lait) incombent à la femme.

L’âge moyen des éleveurs enquêtés est de 47 ans \pm 12 ans. Une grande partie d'entre eux (46\%) n'a reçu aucune forme de scolarisation. Seuls $10 \%$ sont scolarisés (Enseignement général) sous des formes et à des niveaux divers. Le reste est réparti entre l'école coranique et l'alphabétisation. Le ménage éleveur compte en moyenne $7 \pm 5$ actifs.

\section{Caractéristiques communes des systèmes de production laitière}

La race locale la plus répandue dans les systèmes de production de lait du département est le «Zébu Gobra » (95\% des éleveurs enquêtés). Les métisses rencontrées sont issues de croisements entre le Zébu Gobra et des races exotiques (Guzérat et Holstein). Le principal mode de reproduction de ces systèmes est la monte naturelle libre. Le taureau de race exotique est soit loué ou acheté lorsque les moyens financiers le permettent. Si non, le croissement se fait entre les races locales.
La principale source d'aliment est le pâturage naturel. Le système d'élevage est encore extensif avec en moyenne huit (08) heures de conduite des animaux par jour au pâturage. Cependant, les animaux sont souvent complémentés en saison sèche. La complémentation alimentaire concerne surtout les vaches en lactation, celles gestantes, les veaux et celles en état de cachexie avancée. Elle est composée en fonction de l'exploitation de l'aliment de bétails concentré présenté sous forme de granulés, de tourteaux d'arachide, de graines de coton, du son de mil et des résidus de récolte. Ces derniers sont donnés en association ou seul selon des quantités variables en fonction des exploitations, mais pouvant être estimées en moyenne pour l'ensemble des enquêtés à 4,35 $\pm 2,65 \mathrm{~kg}$ /jour/vache.

Sur le plan sanitaire, la vaccination contre les principales maladies et le déparasitage sont effectués par 97,3\% des éleveurs enquêtés. Avec un agent vétérinaire en moyenne par commune et les coûts subventionnés des vaccins, la vaccination et le déparasitage sont des pratiques régulières dans le département.

Les systèmes d'élevage dans le département de Linguère dépendent en majorité de la main d'œuvre familiale (98,2\% des enquêtés). Celle-ci est assurée par le chef du ménage, sa/ses femme(s) et ses enfants. En plus de cette main d'œuvre familiale, certains éleveurs ont recours à la main d'œuvre salariée (39,3\% des enquêtés) pour la conduite des animaux au pâturage. Quant à la traite, la plupart des systèmes le font majoritairement « matin et soir » pour maximiser le revenu tiré de la vente du lait. C'est une activité qui se fait manuellement et nécessite parfois beaucoup de temps en fonction du nombre de vaches en lactation. 
Typologie des systèmes de production laitière

Les deux (02) premières dimensions obtenues après l'Analyse des Correspondances Multiples (ACM) expliquent $26,39 \%$ de la variabilité totale de l'échantillon (Figure 1). La première est celle des paramètres structurels des systèmes de production laitière avec principalement des variables comme le nombre de vaches en lactation par saison (NLSP et NLSS), la distance séparant l'exploitation de l'unité de transformation la plus proche (D2), la production totale de lait en saison des pluies (PSP), l'effectif des femelles du troupeau (FBOV), la production totale de lait en saison sèche (PSS) et le nombre d'actifs du ménage (NA). La seconde est celle des paramètres fonctionnels des systèmes de production laitière avec des variables comme le choix du produit vendu (PV), le mode de payement (MP), le pourcentage de vente par saison (VSP et VSS).

La Classification Ascendante Hiérarchique $(\mathrm{CAH})$ réalisée à partir des coordonnées des observations sur les deux (02) dimensions retenues à partir de l'ACM a fait ressortir trois (03) types de systèmes qui se distinguent essentiellement par la structure $\mathrm{du}$ troupeau et les modalités de fonctionnement $\mathrm{du}$ système de production (Figure 2).

\section{Caractéristiques techniques des systèmes de production laitière}

Les statistiques descriptives et le test de Kruskall Wallis effectuées sur les paramètres techniques des systèmes de production laitière (Tableaux 1 et 2 ) ont montré que le système 1 regroupe des éleveurs caractérisés par des paramètres de structure faibles (Tableau 1). En effet, le nombre d'actifs du ménage, l'effectif des femelles du troupeau et les nombres de vaches en lactation dans chacune des deux (02) saisons y sont les plus faibles des trois (03) systèmes de production (Tableau 1). Les éleveurs des communes de Dahra et de Boulal y sont les plus représentés. En revanche, les éleveurs du système 2 sont caractérisés par des paramètres de structure les plus élevés. Cependant, il n'y a pas de différence significative entre le système 2 et le système 3 quant au nombre d'actifs du ménage.

La production totale de lait des éleveurs du système 1 est la plus faible de l'ensemble des trois (03) systèmes (Tableau 2). Cependant, ils réalisent les meilleures performances sur le plan de la productivité des vaches avec 4,20 $\pm 0,64$ litres/vache/jour et $3,25 \pm 1,01$ litres/vache/jour respectivement en saison pluvieuse et saison sèche. Les plus grandes productions totales sont observées chez les éleveurs du système 2. Par ailleurs, ceux du système 3 ont la plus faible productivité des vaches en lactation. Cette forte production de lait chez les éleveurs du système 2 est essentiellement liée au nombre élevé de vaches en lactation et non à leur productivité.

Les éleveurs du système 1 ayant un accès facile au marché laitier du fait de leur proximité avec les Unités de Transformation Locales (UTL) (en moyenne 6,52 $\pm 2,44 \mathrm{~km}$ ), préfèrent leur vendre le lait frais. Ils ont des taux de vente en saison sèche plus élevés $(58,28 \pm 5,32)$ que ceux du système $2(43,27 \pm$ $5,26)$, situés en moyenne à $27,19 \pm 3,40 \mathrm{~km}$ de l'UTL la plus proche. En saison pluvieuse, il n'y a pas de différence significative entre les taux de vente des systèmes 1 et 2 . En effet, l'éloignement des éleveurs du système 2 des UTL ne constitue pas pour eux un obstacle à la valorisation du lait produit. Ces derniers caillent le lait et ciblent en priorité les marchés hebdomadaires pour la vente. Malgré une orientation plutôt commerciale des éleveurs du système 2 pour la vente du lait, leur objectif premier n'est pas la production 
laitière mais plutôt la production et la vente de bœufs sur pied. Par contre, les éleveurs du système 3 se différencient des 2 autres systèmes par leur orientation exclusive vers l'autoconsommation du lait. En effet, les éleveurs du système 3 ont des taux de vente nuls en saison sèche comme en saison pluvieuse. L'unique objectif de l'élevage est la production et la vente de bœufs sur pied. De plus, ce sont les éleveurs les plus éloignés des UTL.

orientés exclusivement vers étant commercialisation auprès des UTL vendent en majorité le lait frais et sont payés communément sous quinze (15) jours (Non Comptant). En effet, 78,78\% des éleveurs vendant le lait frais et $72,72 \%$ de ceux qui ne sont pas rémunérés au comptant appartiennent au système 1 (Tableau 3). Par contre, 86,84\% et $87,5 \%$ des éleveurs du système 2 sont respectivement payés au comptant et vendent uniquement du lait caillé. L'autoconsommation totale du lait chez les éleveurs du système 3 fait qu'ils n'obtiennent aucune rémunération. Par conséquent, 95,12\% des éleveurs qui ne vendent pas le lait et $95,12 \%$ des éleveurs qui n'ont pas de mode de payement appartiennent à ce système.

\section{Caractéristiques économiques des systèmes} de production laitière

Il ressort des statistiques descriptives et du test de Kruskall Wallis effectués sur les paramètres économiques des systèmes de production laitière (Tableaux 4 et 5) que les charges alimentaires ont été les plus importantes parmi les charges totales de production pour l'ensemble des trois (03) systèmes (plus de 90\%) en saison sèche comme en saison pluvieuse. Au sein des charges alimentaires, le coût moyen d'achat des compléments alimentaires représente le poste de charge le plus important en saison sèche (autour de 90\%) et le plus faible en saison pluvieuse pour chacun des trois (03) systèmes.

Les systèmes 2 et 3 regroupent les éleveurs ayant les coûts de production journaliers les plus élevés en saison pluvieuse (respectivement $817 \pm 366$ F CFA et $340 \pm 46$ F CFA) tandis que les systèmes 1 et 3 , rassemblent les éleveurs ayant de faibles coûts de production journaliers en saison sèche (respectivement $2752 \pm 397$ F CFA et $5108 \pm$ 888 F CFA). Cependant, il n'y a pas de différence significative entre le coût de production journalier par vache en saison sèche pour les éleveurs des trois systèmes (Tableau 4).

En saison sèche, ce sont les systèmes 1 et 2 qui regroupent les éleveurs ayant les chiffres d'affaires journaliers les plus élevés $(1811 \pm 264$ et $3420 \pm 649)$. Etant donné que les éleveurs du système 3 ne vendent par le lait produit, nous n'en ferons pas cas dans cette rubrique. Nous considérons donc qu'ils ont des chiffres d'affaire et des marges nulles. Les marges brutes journalières de ces deux (02) systèmes de production laitière sont négatives $(-940 \pm 393$ et $-4009 \pm 1029)$. Mais elles sont plus prononcées au niveau du système 2 (Tableau 5). En revanche, en saison pluvieuse, les éleveurs du système 2 ont des chiffres d'affaire plus élevés que ceux du système $1(3128 \pm 397$ et $7348 \pm 984)$. Quant aux marges brutes journalières, elles sont positives et plus élevées au niveau du système 2. Cependant, les éleveurs du système 1 réalisent de meilleures performances en matière de productivité avec des marges brutes journalières par vache $(1047 \pm 165)$ plus importantes que celles des éleveurs du système $2(700 \pm 66$, Tableau 5$)$. 


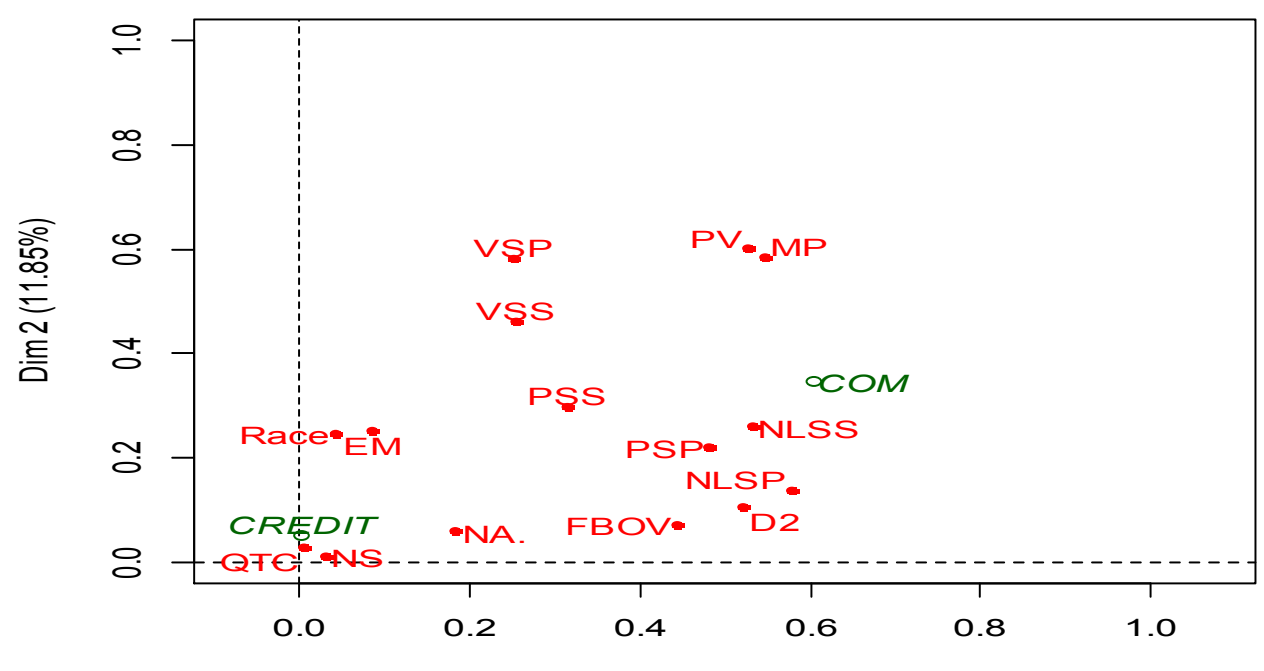

$\operatorname{Dim} 1(14.53 \%)$

Figure 1 : Projection des variables dans le plan formé par les deux premières dimensions de l'ACM. $\mathrm{COM}=$ Communes, CREDIT $=$ Bénéficiaire de prêt bancaire ou non, D2 = Distance qui sépare l'exploitation de l'unité de transformation la plus proche, $\mathrm{EM}=$ Effectif des métisses, $\mathrm{FBOV}=$ Effectif des femelles du troupeau, $\mathrm{MP}=$ Mode de Payement, NA. $=$ Nombre d'actifs du ménage, NLSP $=$ Nombre de vaches en lactation en saison des pluies, NLSS $=$ Nombre de vaches en lactation en saison sèche, NS = Nature du système de production, PSP = Production totale de lait en saison des pluies, PSS $=$ Production totale de lait en saison sèche, $\mathrm{PV}=$ produit vendu, $\mathrm{QTC}=$ Quantité totale de compléments alimentaires, VSP = Pourcentage de lait vendu en saison des pluies, VSS = Pourcentage de lait vendu en saison sèche. Les variables COM et CREDIT ont été pris comme des variables qualitatives supplémentaires.



$\operatorname{Dim} 1(14.53 \%)$

Figure 2: Projection des observations dans le plan formé par les deux premières dimensions de l'ACM. 
Tableau 1: Caractérisation des systèmes de production laitière suivant les paramètres de structuration.

\begin{tabular}{lcccc}
\hline Variable & Système 1 & Système 2 & Système 3 & Probabilité \\
\hline N. d'actifs ménage & $4 \pm 1 \mathrm{~b}$ & $8 \pm 1 \mathrm{a}$ & $9 \pm 1 \mathrm{a}$ & 0,00 \\
Eff. des femelles & $11 \pm 2 \mathrm{c}$ & $56 \pm 6 \mathrm{a}$ & $35 \pm 5 \mathrm{~b}$ & 0,00 \\
N. de vaches en lactation SP & $3 \pm 1 \mathrm{c}$ & $10 \pm 1 \mathrm{a}$ & $8 \pm 1 \mathrm{~b}$ & 0,00 \\
N. de vaches en lactation SS & $3 \pm 1 \mathrm{c}$ & $9 \pm 1 \mathrm{a}$ & $7 \pm 1 \mathrm{~b}$ & 0,00 \\
Commune & Dahra/Boulal & Yang-yang & Lagbar & \\
\hline
\end{tabular}

N. = Nombre ; Eff = Effectif ; SP = Saison Pluvieuse ; SS = Saison Sèche. Les lettres différentes sur la même ligne montrent une différence significative entre les systèmes au seuil de $5 \%$ selon le test de Kruskall Wallis.

Tableau 2: Caractérisation des systèmes de production laitière suivant les paramètres de production et de valorisation du lait.

\begin{tabular}{lcccc}
\hline Variable & Système 1 & Système 2 & Système 3 & Probabilité \\
\hline $\begin{array}{l}\text { Quantité produite SP } \\
\text { (L/Ferme/Jr) }\end{array}$ & $14,88 \pm 2,72 \mathrm{c}$ & $29,03 \pm 3,2 \mathrm{a}$ & $20,16 \pm 2,54 \mathrm{~b}$ & 0,0000 \\
$\begin{array}{l}\text { Quantité produite SS } \\
\text { (L/Ferme/Jr) }\end{array}$ & $6,79 \pm 0,82 \mathrm{~b}$ & $13,94 \pm 2,2 \mathrm{a}$ & $7,35 \pm 0,98 \mathrm{~b}$ & 0,001 \\
$\begin{array}{l}\text { Quantité produite SP } \\
\text { (L/Vache/Jr) }\end{array}$ & $4,20 \pm 0,64 \mathrm{a}$ & $3,08 \pm 0,25 \mathrm{ab}$ & $2,58 \pm 0,19 \mathrm{~b}$ & 0,025 \\
$\begin{array}{l}\text { Quantité produite SS } \\
\text { (L/Vache/Jr) }\end{array}$ & $3,25 \pm 1,01 \mathrm{a}$ & $1,56 \pm 0,23 \mathrm{ab}$ & $1,22 \pm 0,15 \mathrm{~b}$ & 0,001 \\
$\begin{array}{l}\text { Distance Ferme }-\mathrm{UTL}(\mathrm{km}) \\
\text { Taux de vente SP }(\%)\end{array}$ & $6,52 \pm 2,44 \mathrm{c}$ & $27,19 \pm 3,40 \mathrm{~b}$ & $39,63 \pm 4,05 \mathrm{a}$ & 0,0000 \\
$\begin{array}{l}\text { Taux de vente SS }(\%) \\
\text { Orientation }\end{array}$ & $61,26 \pm 5,09 \mathrm{a}$ & $61,90 \pm 3,69 \mathrm{a}$ & $0 \mathrm{~b}$ & 0,0000 \\
\hline
\end{tabular}

$\mathrm{L}=$ Litre $; \mathrm{Jr}=$ Jour ; SS = Saison Sèche ; SP = Saison Pluvieuse ; UTL = Unité de Transformation Locale. Les lettres
différentes sur la même ligne montrent une différence significative entre les systèmes au seuil de 5\% selon le test de Kruskall Wallis.

Tableau 3 : Caractérisation des systèmes de production laitière suivant le mode de payement et le produit vendu.

\begin{tabular}{lcc}
\hline & Système 1 & \\
\hline Modalité & Cla/Mod & P.value \\
\hline $\mathrm{PV}=\mathrm{LF}$ & 78,78 & 0,00 \\
$\mathrm{MP}=\mathrm{CP} \mathrm{NP}$ & 86,36 & 0,00 \\
$\mathrm{MP}=\mathrm{NCP}$ & 72,72 & 0,00 \\
\hline \multicolumn{3}{c}{ Système 2 } \\
\hline Modalité & Cla/Mod & P.value \\
\hline $\mathrm{MP}=\mathrm{CP}$ & 86,84 & 0,00 \\
$\mathrm{PV}=\mathrm{LC}$ & 87,5 & 0,00 \\
\hline & Système 3 \\
\hline Modalité & Cla/Mod & P.value \\
\hline $\mathrm{MP}=\mathrm{MPR}$ & 95,12 & 0,00 \\
$\mathrm{PV}=\mathrm{PVR}$ & 95,12 & 0,00 \\
\hline
\end{tabular}

$\mathrm{Cla}=$ Classe $;$ Mod $=$ Mode $;$ P.value $=$ Probabilité $; \mathrm{MP}=$ Mode de payement de l'UTL la plus proche $; \mathrm{MPR}=$ Pas de mode de payement ; PV = Produit vendu ; PVR = Pas de produit vendu ; $\mathrm{LC}=$ Lait caillé ; LF = Lait frais ; $\mathrm{CP}=$ Comptant ; NCP $=$ Non Comptant $; \mathrm{CP} \_\mathrm{NP}=$ Comptant et Non Comptant. 
Tableau 4 : Caractérisation des systèmes de production laitière suivant différentes charges.

\begin{tabular}{lcccc}
\hline Variable & Système 1 & Système 2 & Système 3 & Probabilité \\
\hline CA/CP_SP (\%) & $93,63 \pm 1,15$ & $93,36 \pm 1,03$ & $90,27 \pm 2,48$ & 0,56 \\
CA/CP_SS (\%) & $99,41 \pm 0,15$ & $99,27 \pm 0,18$ & $98,78 \pm 0,44$ & 0,72 \\
CCA/CA_SP (\%) & $2,49 \pm 2,49$ & $0,65 \pm 0,65$ & 0 & 0,57 \\
CCA/CA_SS (\%) & $93,14 \pm 0,70$ & $90,64 \pm 1,5$ & $86,41 \pm 4,12$ & 0,99 \\
CP_SP (F CFA/Jr/Ferme) & $179 \pm 20 \mathrm{~b}$ & $817 \pm 366 \mathrm{a}$ & $340 \pm 46 \mathrm{a}$ & 0,00 \\
CP_SS (F CFA/Jr/Ferme) & $2752 \pm 397 \mathrm{~b}$ & $7429 \pm 1084 \mathrm{a}$ & $5108 \pm 888 \mathrm{~b}$ & 0,00 \\
CP_SP (F CFA/Jr/Vache) & $65 \pm 14 \mathrm{a}$ & $78 \pm 31 \mathrm{~b}$ & $46 \pm 5 \mathrm{ab}$ & 0,02 \\
CP_SS (F CFA/Jr/Vache) & $898 \pm 90 \mathrm{a}$ & $820 \pm 89 \mathrm{a}$ & $808 \pm 98 \mathrm{a}$ & 0,61 \\
\hline
\end{tabular}

$\mathrm{CA}=$ Charges Alimentaires ; $\mathrm{CP}=$ Coût de Production ; CCA = Coût d'achat des Compléments Alimentaires ; Jr = Jour ; SS = Saison Sèche $; \mathrm{SP}=$ Saison Pluvieuse $; \mathrm{F}$ CFA = Francs CFA. Les lettres différentes sur la même ligne montrent une différence significative entre les systèmes au seuil de 5\% selon le test de Kruskall Wallis.

Tableau 5 : Caractérisation des systèmes de production laitière suivant leurs rentabilités.

\begin{tabular}{lcccc}
\hline Variable & Système 1 & Système 2 & Système 3 & Probabilité \\
\hline CAff_SS (F CFA/Jr/Ferme) & $1811 \pm 264 \mathrm{a}$ & $3420 \pm 649 \mathrm{a}$ & $0 \mathrm{~b}$ & 0,00 \\
MB_SS (F CFA/Jr/Ferme) & $-940 \pm 393 \mathrm{a}$ & $-4009 \pm 1029 \mathrm{~b}$ & - & 0,00 \\
MB_SS (F CFA/Jr/Vache) & $-188 \pm 183 \mathrm{a}$ & $-418 \pm 93 \mathrm{a}$ & - & 0,00 \\
CAff_SP (F CFA/Jr/Ferme) & $3128 \pm 397 \mathrm{~b}$ & $7348 \pm 984 \mathrm{a}$ & - & 0,00 \\
MB_SP (F CFA/Jr/Ferme) & $2949 \pm 385 \mathrm{~b}$ & $6531 \pm 757 \mathrm{a}$ & - & 0,00 \\
MB_SP (F CFA/Jr/Vache) & $1047 \pm 165 \mathrm{a}$ & $700 \pm 66 \mathrm{~b}$ & - & 0,00
\end{tabular}

$\mathrm{CP}=$ Coût de Production ; SS = Saison Sèche ; SP = Saison Pluvieuse ; CAff = Chiffre d'Affaire ; MB = Marge Brute. . Les lettres différentes sur la même ligne montrent une différence significative entre les systèmes au seuil de $5 \%$ selon le test de Kruskall Wallis.

\section{DISCUSSION}

Les résultats ont permis d'identifier trois (03) systèmes de production laitière dans le département de Linguère qui se distinguent par l'effectif des femelles du troupeau, le nombre de vaches en lactation et la production laitière. En effet, les faibles ressources pastorales (surface de pâturage naturel) disponibles dans les zones urbaines obligent les éleveurs du système 1 situés à la périphérie des villes à réduire leurs cheptels contrairement aux éleveurs situés dans les zones rurales (systèmes 2 et 3 ). Cette conclusion est corroborée par l'étude de YapiGnaoré et al. (2009) qui ont observé de petits cheptels chez des éleveurs localisés dans la périphérie d'Abidjan en Côte d'Ivoire. La conséquence immédiate de la faiblesse des actifs productifs dans certaines communes de la zone d'étude est le faible niveau de production laitière journalière des exploitations. Cependant, on observe une 
grande disparité entre les systèmes du point de vue de la productivité des vaches en lactation. Ndiaye et al. (2014) ont trouvé des résultats similaires dans des systèmes de production laitière au Sénégal où le premier groupe typologique a une production de lait journalière par vache entre 2 et 4 litres ou même supérieure (comparable aux éleveurs des systèmes 1 et 2 à Linguère) et le second, une production journalière par vache inférieure à 2 litres (comparable aux éleveurs du système 3 ). En outre, les résultats de la présente étude sont proches de ceux obtenus par Assani et al. (2015), Youssao (2015), Bouyer (2006), N'diaye (2006) et Cissé (2000) en Afrique de l'Ouest sur des bovins de race «Zébu » en élevage extensif dont la production varie entre 1,52 à 4,7 litres/vache/jour.

D'après Bonfoh et al. (2007), Asseu (2010) et Ndione et al. (2014), la supplémentation des vaches en lactation en saison sèche permettrait d'augmenter substantiellement leurs productions laitières. Cependant, la quantité de compléments alimentaires distribuée n'est pas très variable d'un système à l'autre à Linguère $(4,33 \pm 2,65$ $\mathrm{kg} /$ vache/jour) et inférieure à celle rapportée par Si-Tayeb et al. (2014) en Algérie qui est de $9 \pm 3 \mathrm{~kg} /$ vache/jour dans des systèmes orientés vers la production laitière. Ainsi, les pratiques alimentaires expliquent en partie la productivité des vaches laitières du département de Linguère. Toutefois, il est nécessaire de souligner que les objectifs de production ne sont pas les mêmes et que l'élevage est encore allaitant dans les systèmes de production laitière au Sénégal.

La distance séparant l'exploitation de l'unité de transformation locale (UTL) la plus proche, le choix du produit vendu par le producteur et le pourcentage de vente qui reflète l'orientation commerciale ou non constituent d'autres caractéristiques très discriminantes des systèmes de production laitière de Linguère. En effet, d'après les résultats, la distance séparant l'exploitation de l'UTL la plus proche semble déterminer le choix $\mathrm{du}$ produit vendu et l'orientation commerciale ou non du système de production laitière. Yapi-Gnaoré et al. (2009) ont observé chez des fermes de production laitière dans le Sud de la Côte d'Ivoire, des caractéristiques similaires du point de vue de leurs localisations et de leurs orientations commerciales. Ainsi, les UTL doivent développer des mécanismes de collecte permettant de réduire la distance à parcourir par le producteur pour la vente du lait. Néanmoins, ces résultats ont besoin d'être approfondis vue l'importance actuelle des UTL dans l'offre de produits laitiers aux consommateurs urbains.

Les résultats de la présente étude montrent également que les éleveurs de Linguère se distinguent par l'objectif visé par l'élevage. Ainsi, trois (03) systèmes de production laitière se distinguent comme cela a été le cas en zones montagneuses d'Algérie et dans la zone soudanienne du Bénin (Youssao et al. 2013 ; Mouhous, 2014). Ces caractéristiques illustrent bien ceux des producteurs de lait de l'Afrique de l'Ouest qui ne se sont pas spécialisés dans la production laitière (Corniaux et al., 2012). Selon ces auteurs, " $\mathrm{Au}$ fond, le lait est davantage un coproduit qu'une fin en soi ».

Sur le plan économique, la diversité des systèmes de production laitière de Linguère permet de comprendre leur rentabilité. En effet, la productivité des vaches en lactation et le prix de vente plus rémunérateur au niveau des unités de transformation locales permettent aux éleveurs du système 1 de réaliser de meilleurs profits par rapport aux éleveurs du système 2 en saison sèche comme en saison pluvieuse. Ces deux (02) systèmes réalisent des pertes en saison sèche du fait des fortes dépenses en compléments alimentaires et de la faible 
productivité des vaches laitières dans cette période. En revanche, ils réalisent des profits en saison pluvieuse à cause de l'absence de la complémentation.

\section{Conclusion}

La présente étude à identifier trois (03) systèmes de production laitière à Linguère. Ils se distinguent principalement par les modalités de structuration du troupeau et de fonctionnement $\mathrm{du}$ système laitier. Les variables comme le nombre de femelles en lactation, le nombre d'actifs du ménage, la production laitière journalière, le produit vendu et le mode de payement ont été très discriminantes. Les modalités de valorisation $\mathrm{du}$ lait produit et l'orientation commerciale ou non permettent de mieux comprendre les performances économiques des différents systèmes de production laitière du département de Linguère. La valorisation du lait à l'image de celle des producteurs du système 1 à travers les unités de transformation locales (mini-laiteries et centres de collecte) peut permettre la promotion de l'élevage périurbain. Ainsi, le développement de la production laitière au Sénégal pourrait passer par un meilleur appui aux éleveurs des systèmes 1 et 2 , par exemple en nouant des partenariats avec les systèmes financiers décentralisés pour des octrois de crédit aux producteurs. Ainsi, ces producteurs pourront maintenir par moment les vaches en stabulation en saison sèche pour une meilleure production laitière. En outre, les unités de transformation locales doivent organiser la collecte du lait frais sur la base de la distance qui sépare les fermes de production des unités de transformation locales et de l'orientation commerciale des différents systèmes identifiés.

\section{CONFLIT D'INTERETS}

Les auteurs déclarent qu'il n'y a aucun conflit d'intérêts.

\section{CONTRIBUTIONS DES AUTEURS}

Ce travail a été réalisé en collaboration entre tous les auteurs. SSD et IW ont conçu l'étude, ont écrit le protocole et ont fourni une revue approfondie de la littérature et des implications politiques et économiques des résultats de l'étude. SSD a collecté les données et écrit le premier brouillon du manuscrit. CEA et SSD ont effectué les analyses statistiques. IW et CEA ont supervisé l'ensemble du travail et examiné toutes les ébauches du manuscrit. Tous les auteurs ont lu et approuvé le manuscrit final.

\section{REMERCIEMENTS}

Ce travail a été financé par l'Organisation des Nations Unies pour le Développement Industriel (ONUDI) dans le cadre du projet de promotion du développement économique local de la région de Louga. Les principaux instituts ayant aidés à la réalisation de ce travail sont l'Ecole Nationale Supérieure d'Agriculture de Thiès de l'Université de Thiès et le Centre de Recherches Zootechniques de Dahra. Les auteurs remercient les éleveurs et les agents vétérinaires pour leur collaboration.

\section{REFERENCES}

Agence Nationale de la Statistique et de la Démographie (ANSD). 2010. Situation économique et sociale de la région de Louga, p. 89.

Agence Régionale de Développement (ARD) de Louga. 2013. Rapport de la conférence d'harmonisation, p. 34.

Assani SA, Assogba B, Toukourou Y, Alkoiret IT. 2015. Productivity of Gudali cattle farms located in the commons of Malancity and Karimama extreme north of Benin. LRRD, 27(7): 1-9. 
Asseu CK, 2010. Evaluation du degré d'acceptation de l'insémination artificielle bovine à Kaolack au Sénégal. Thèse de Médecine Vétérinaire, Ecole Inter Etats des Sciences et Médecine Vétérinaires de Dakar, Université Cheick Anta Diop de Dakar, Dakar, Sénégal, p. 92.

Bonfoh B, Fokou G, Ould Taleb M, Fané A, Woirin D, Laimaibao N, Zinsstag J. 2007. Dynamiques des systèmes de production laitière, risques et transformations socioconomiques au Mali. Rev. Elev. Méd. Vét. Pays Trop., 60(1-4): 67-76.

Bouyer B. 2006. Bilan et analyse de l'utilisation de l'insémination artificielle dans les programmes d'amélioration génétique des races laitières en Afrique soudano-sahélienne. Thèse de Doctorat en Médecine Vétérinaire, Ecole Nationale Vétérinaire de Lyon, Université Claude Bernard - Lyon I, Lyon, France, p.107.

Cissé S. 2000. Stratégie nationale en matière de diversité biologique. Tome 1: situation générale de la diversité biologique au Mali, p.122.

Corniaux C. 2015. Voyage au pays du lait : Dynamiques laitières dans le monde. In Dynamique des Bassins Laitiers Entre Globalisation et Territorialisation, Napoléone M, Corniaux C, Leclerc B (eds). Inra-Sad : Paris ; 312-320.

Corniaux C, Alary V, Gautier D, Duteurtre G. 2012. Producteur laitier en Afrique de l'Ouest : une modernité rêvée par les techniciens à l'épreuve du terrain. Autrepart., 3(62): 17-36. DOI : $10.3917 /$ autr.062.0017

Dia D. 2009. Les territoires d'élevage à l'épreuve des dynamiques politiques et économiques : éléments pour une géographie du lait au Sénégal. Thèse de
Doctorat de troisième cycle, Université Cheikh Anta DIOP, Dakar, p. 336.

Dia D. 2013. Etude relative à la formulation du programme d'actions détaillé de développement de la filière lait en zone UEMOA, p. 40

Direction de la Prévision et des Etudes Economiques (DPEE). 2011. Situation économique et financière en 2011 et perspectives en 2012, p. 11.

Duteurtre V. 2006. État des lieux de la filière lait et produits laitiers au Sénégal. 98p. Disponible sur http://www.agroalimentaire.sn/IMG/pdf/ Filiere_lait_Senegal_2005_VF.pdf

Faihun AML, Akouedegni CG, Olounlade PA, Adenile DA, Hounzangbe-Adote SM. 2017. Typologie des élevages de cobayes (Cavia porcellus) au Bénin. Int. J. Biol. Chem. Sci., 11(2): 556-570. DOI : https://dx.doi.org/10.4314/ijbcs.v11i2.3

FAOSTAT, 2014. www.fao.org, Consulté en Février 2017

Goodman LA. 1961. Snowball Sampling. Annals of Mathematical Statistics, 32:148-170. Disponible sur https://projecteuclid.org/download/pdf_1 /euclid.aoms/1177705148 (Consulter le février 2017)

Holloway G, Nicholson C, Delgado C, Staal S, Ehui S. 2000. Agroindustrialization through institutional innovation. Transaction costs, cooperatives and milk market development in the east-African highlands. Agricultural Economics, 23: 279-288. Disponible sur http://ageconsearch.umn.edu/bitstream/1 76215/2/agec2000v023i003a008.pdf (Consulter le 15 février 2017)

Husson F, Lê S, Pagês G. 2010. Exploratory Multivariate Analysis by Exemple, Using R. (1st Edn). Press, Taylor and Francis Group.

Kassa SK, Ahounou S, Dayo GK, Salifou C, Issifou TM, Dotché I, Gandonou SP, 
Yapi Gnaoré V, Koutinhouin B, Mensah GA, Youssao I. 2016. Performances de production laitière des races bovines de l'Afrique de l'Ouest. Int. J. Biol. Chem. Sci., $\quad$ 10(5): 2316-2330. http://dx.doi.org/10.4314/ijbcs.v10i5.29

Marshall K, Tebug S, Juga J, Tapio M, Missohou A. 2016. De meilleures races laitières bovines et une meilleure gestion en faveur des populations pauvres rurales au Sénégal. ILRI Research Brief, 69 : 16. Disponible sur https://cgspace.cgiar.org/handle/10568/7 5979 (Consulté le février 2017)

Mbetid-Bessane E, Havard M, Djamen NP, Djonnewa A, Djondang $\mathrm{K}$, Leroy J. 2002. Typologies des exploitations agricoles dans les savanes d'Afrique centrale. Actes du colloque, 27-31 mai 2002, Garoua, Cameroun, p. 10.

Mouhous A, Alary V, Huguenin J. 2014. Stratégies d'adaptation des éleveurs bovins laitiers en zone montagneuse d'Algérie. Revue d'Elevage et de Médecine Vétérinaire des Pays Tropicaux, 67(4): 193-200.

N'Diaye A. 2006. Le lait dans les stratégies de diversification des revenus des agropasteurs de la région de Fatick. Mémoire d'Ingénieur Agronome, Département d'Economie et Sociologie Rurales, Ecole Nationale Supérieure d'Agriculture, Sénégal, p. 93.

Ndiaye NP, Sow A, Ndiaye S, Sembene M, Sawadogo GJ. 2014. Phenotypical characterization of senegalese local cattle breeds using multivariate analysis. J. Anim. Vet. Adv., 13(20): 1150-1159.

Ndione FC, Agbangba EC, Ndiaye O, Sane I, Diouf A, Diop AT. 2014. Effects of protein and mineral supplementation on the performance of lactating dairy cows gobra and crossbreed guzerat during the dry season in Senegal. Journal of Animal Sciences, 4(1): 18-22. Disponible sur http://dx.doi.org/10.4236/ojas.2014.4100 3

Pinaud S. 2014. La poudre de lait, le trader parisien et le commerçant bamakois. Une sociologie économique de la mondialisation. Thèse de doctorat en sociologie, Université de Paris Ouest Nanterre, Paris, p. 570.

Si-Tayeb H, Mouhous A, Cherfaoui LM. 2014. Caractérisation de l'élevage bovin laitier en Algérie : cas de la zone de Fréha à Tizi-Ouzou, p. 12. http://www.journees3r.fr/IMG/pdf/Texte _21_systemes_A-Mouhous.pdf

Yapi-Gnaoré CV, N'Goran KE, Fantodji A, Ahoussou N. 2009. Influence des facteurs de production sur l'élevage laitier périurbain des régions de savane et de forêt de Côte d'Ivoire. J. Appl. Biosci., 19: 10651073. www.biosciences.elewa.org

Youssao AKI, Dahouda M, Attakpa EY, Koutinhouin GB, Ahounou GS, Toleba SS, Balogoun BS. 2013. Diversité des systèmes d'élevages de bovins de race bovine Borgou dans la zone soudanienne du Bénin. Int. J. Biol. Chem. Sci., 7(1): 125-146.

DOI: http://dx.doi.org/10.4314/ijbcs.v7i1i.11

Youssao AKI. 2015. Programme National d'Amélioration Génétique. Projet d'Appui aux Filières Lait et Viande (PAFILAV). Cotonou, Bénin, p. 344. 